\title{
Nanostructured Mesoporous Silica Wires with Intrawire Lamellae via Evaporation-Induced Self-Assembly in Space-Confined Channels
}

\author{
Michael Z. Hu, ${ }^{1}$ Donglu Shi, ${ }^{2}$ and Douglas A. Blom ${ }^{3}$ \\ ${ }^{1}$ Energy and Transportation Science Division, Oak Ridge National Laboratory, Oak Ridge, TN 37831-6181, USA \\ ${ }^{2}$ The Materials Science and Engineering Program, Department of Mechanical and Materials Engineering, University of Cincinnati, \\ Cincinnati, OH 45221-0072, USA \\ ${ }^{3}$ University of South Carolina, 1212 Greene Street, Columbia, SC 29208, USA
}

Correspondence should be addressed to Michael Z. Hu; hum1@ornl.gov

Received 24 January 2014; Accepted 22 March 2014; Published 6 April 2014

Academic Editor: Sayan Bhattacharyya

Copyright (C) 2014 Michael Z. Hu et al. This is an open access article distributed under the Creative Commons Attribution License, which permits unrestricted use, distribution, and reproduction in any medium, provided the original work is properly cited.

\begin{abstract}
Evaporation-induced self-assembly (EISA) of silica sol-gel ethanol-water solution mixtures with block-copolymer were studied inside uniform micro/nano-channels. Nanostructured mesoporous silica wires, with various intrawire self-assembly structures including lamellae, were prepared via EISA process but in space-confined channels with the diameter ranging from $50 \mathrm{~nm}$ to $200 \mathrm{~nm}$. Membranes made of anodized aluminum oxide (AAO) and track-etched polycarbonate (EPC) were utilized as the arrays of space-confined channels (i.e., 50, 100, and $200 \mathrm{~nm}$ EPC and $200 \mathrm{~nm}$ AAO) for infiltration and drying of mixture solutions; these substrate membranes were submerged in mixture solutions consisting of a silica precursor, a structure-directing agent, ethanol, and water. After the substrate channels were filled with the solution under vacuum impregnation, the membrane was removed from the solution and dried in air. The silica precursor used was tetraethyl othosilicate (TEOS), and the structure-directing agent employed was triblock copolymer Pluronic-123 (P123). It was found that the formation of the mesoporous nanostructures in silica wires within uniform channels were significantly affected by the synthesis conditions including (1) preassemble TEOS aging time, (2) the evaporation rate during the vacuum impregnation, and (3) the air-dry temperature. The obtained intrawire structures, including 2D hexagonal rods and lamellae, were studied by scanning transmission electron microscopy (STEM). A steric hindrance effect seems to explain well the observed polymer-silica mesophase formation tailored by TEOS aging time. The evaporation effect, airdrying effect, and AAO versus EPC substrate effect on the mesoporous structure of the formed silica wires were also presented and discussed.
\end{abstract}

\section{Introduction}

Nanostructured porous silica has numerous applications, such as in the area of catalysis, adsorption, molecular separation, and templating in the syntheses of a variety of inorganic wires $[1,2]$. In bulk phase of solutions, there have been extensive investigations on the synthetic parameters and processing conditions affecting the architecture of the selfassembled porous domains. The research has been focused on identifying the fundamental self-assembly mechanisms involving both inorganic components and organic surfactants as structure-directing agents. One of the mechanisms proposed is the so-called nanocasting [3, 4], in which solgel syntheses of inorganic solids take place in ordered environments formed by the self-assembly of organic surfactants. Another mechanism, called cooperative self-assembly [5], is based on the idea that the inorganic precursors and organic surfactants are self-assembled simultaneously. The final selfassembled nanostructures are affected by the amount of the inorganic precursor and the surfactant, as well as many other synthetic process parameters.

The cooperative organic-inorganic self-assembly is a unique approach to synthesize nanostructured silica with various porous structures and morphologies. In the past 
ten years, amphiphilic block copolymers, such as diblock polystyrene-block-poly(ethylene oxide) (PS-b-PEO) and triblock poly(ethylene oxide)-block-poly(propylene oxide)block-poly(ethylene oxide) (PEO- $b$-PPO- $b$-PEO), have been used as soft structure-directing agents in the synthesis of nanostructured porous inorganic solids with controlled porous structures and morphologies [5]. Microphase separation phenomena during evaporation of triblock copolymer-solvent solutions could generate various mesoscale phases and patterns in gelled/dried polymer. The polymeric microphase evolution can serve as the fundamental templating mechanism for guiding the selfassembly formation of inorganic structures if the sol-gel inorganic reactant precursors are mixed homogeneously with the block copolymer. In fact, sol-gel reaction occurs to precipitate out inorganic solids while the polymeric microphase evolves. During evaporative drying process, the precipitated inorganic solid distributes selectively in one of the two polymer phases in the microphase pattern, one of which phases is hydrophobic and the other is hydrophilic. After burning off the polymer, the remained inorganic solids take the self-assembled mesoporous structures that correspond to the polymer microphase pattern.

There have been some literature reports on the synthesis of nanostructured mesoporous silica wires by the selfassembly of silica precursors with the triblock copolymer or surfactant inside space-confined channels of substrate membranes such as anodized aluminum oxide (AAO) [5-9]. We have previously reported the STEM study of mesoporous silica wire formation inside the channels of AAO membranes [9]. Our previously reported nanostructured mesoporous silica wires have intrawire structures of only hexagonallyarranged cylindrical mesophases [9]. For those silica wires prepared in the AAO channel, two orientations of the cylindrical pores were reported $[6,9]$ : one had a columnar orientation with the long axes of cylindrical nanopores aligned along the long axes of the AAO channel and the other had circular orientation with the cylindrical nanopores aligned in the axial direction of the AAO channel. These intrawire nanostructures depend sensitively on the silicato-surfactant ratio [10], the humidity condition of the solgel process [11], the concentration of the surfactant [12], and the synthetic temperature [13]. For those self-assembled copolymer-silica wires made in the $400 \mathrm{~nm}$ track-etched polycarbonate membranes (EPC) channels, the parallelpacked 2D hexagonal cylinders were perpendicular to the wire axis; the copolymer used was $\mathrm{PEO}(20)-b-\mathrm{PPO}(70)-b$ $\mathrm{PEO}(20)$ (Pluronic, P123) [14]. No mesoporous silica wires with intrawire nanostructure of lamellae were reported. In this paper, we utilize the smaller EPC channels to provide space confined channels $(50,100$, and $200 \mathrm{~nm})$ as a study tool and a $200 \mathrm{~nm}$ AAO to see the effect of substrate characteristic.

Here, we report scanning transmission electron microscopy (STEM) results of a novel intrawire nanoporous structure, with its lamellar discs perpendicular to the wire axis. The porous silica wire with such a novel nanostructure was prepared in the space-confined EPC channel by evaporation-induced self-assembly with P123 as the structure-directing agent. Experimental results on structural dependence on various processing conditions are also reported here in this paper. Instead of the $400 \mathrm{~nm}$ channel diameter [14], this work used the EPC membranes of smaller channel diameters ranging from $50 \mathrm{~nm}$ to $200 \mathrm{~nm}$. To investigate the structural dependence on the substrate, AAO membranes were also used with channel diameters on the order of $200 \mathrm{~nm}$. These substrate membranes were immersed in a solution consisting of a silica precursor, a structure-directing agent, ethanol, and water. Vacuum impregnation, as described in our previous paper [9], was performed to fill the substrate channels with the mixture solution. After impregnation, the membrane was removed and dried in air, followed by calcination to obtain the porous silica wires with intrawire nanostructures of cylinders or lamellae. Tetraethyl orthosilicate (TEOS) was used as the silica precursor while triblock copolymer Pluronic-123 (P123) was the structure-directing mesophase-forming agent. Both synthesis and processing conditions were varied to fine-tune the intrawire nanoporous structures. These include preassembled TEOS aging time, the evaporation rate during the vacuum impregnation, and the air-dry temperature. STEM was used to study the nanostructures prepared including $2 \mathrm{D}$ hexagonal rods and lamellae. The nanostructured mesoporous silica wires with intrawire lamellar disc perpendicular to the wire axis is reported in this paper.

\section{Experimental Section}

2.1. Materials. All chemicals were used as received. Silica precursor $\mathrm{Si}\left(\mathrm{OC}_{2} \mathrm{H}_{5}\right)_{4}$ (i.e., TEOS) was from J. T. Baker Inc., and absolute ethanol $\left(\mathrm{C}_{2} \mathrm{H}_{5} \mathrm{OH}\right.$ or EtOH) was from AAPER Alcohol Chemical Co. (absolute-200 proof). Hydrochloric acid $(\mathrm{HCl})(6.000 \pm 0.030 \mathrm{~N})$ was provided by VWR, and aqueous solution of $\mathrm{HCl}$ with $\mathrm{pH}$ of 2.00 was prepared by diluting $\mathrm{HCl}(6.000 \pm 0.030 \mathrm{~N})$ with deionized water. The $\mathrm{pH}$ of the solution was regulated via a $\mathrm{pH}$ meter. Triblock copolymer PEO(20)- $b$ - $\mathrm{PPO}(70)-b-\mathrm{PEO}$ (20) (Pluronic, P123), as a mesophase-forming "soft template" (i.e., polymeric microphases), was from BASF Corporation. AAO and EPC membranes, in forms of micro/nano scale channels, were used as "hard templates" (i.e., array of uniform cylindrical, parallel tubular channels) to confine the cooperative selfassembly of P123 and the silica precursor. The AAO membrane (Anodisc 13) with channels around $200 \mathrm{~nm}$ in diameter was purchased from WHATMAN, while the PVP-treated standard white membrane EPC with channels ranging from $50 \mathrm{~nm}$ to $200 \mathrm{~nm}$ in diameter was from SPI SUPPLIES.

2.2. Synthesis. Nanostructured mesophased P123-silica composite wires were prepared inside the uniform channels of AAO or EPC membranes via evaporation-induced selfassembly. Removal of EPC (by calcination at $60^{\circ} \mathrm{C} / \mathrm{h}$ to $550^{\circ} \mathrm{C}$ for $5 \mathrm{~h}$ ) or removal of $\mathrm{AAO}$ (by dissolution in $5 \mathrm{M} \mathrm{HCl}$ at $60^{\circ} \mathrm{C}$ for $1 \mathrm{~h}$ ) results in architectured mesoporous wires. The formation of these nanowires by the sol-gel and polymer microphase forming process was space-confined within EPC or AAO channels. 


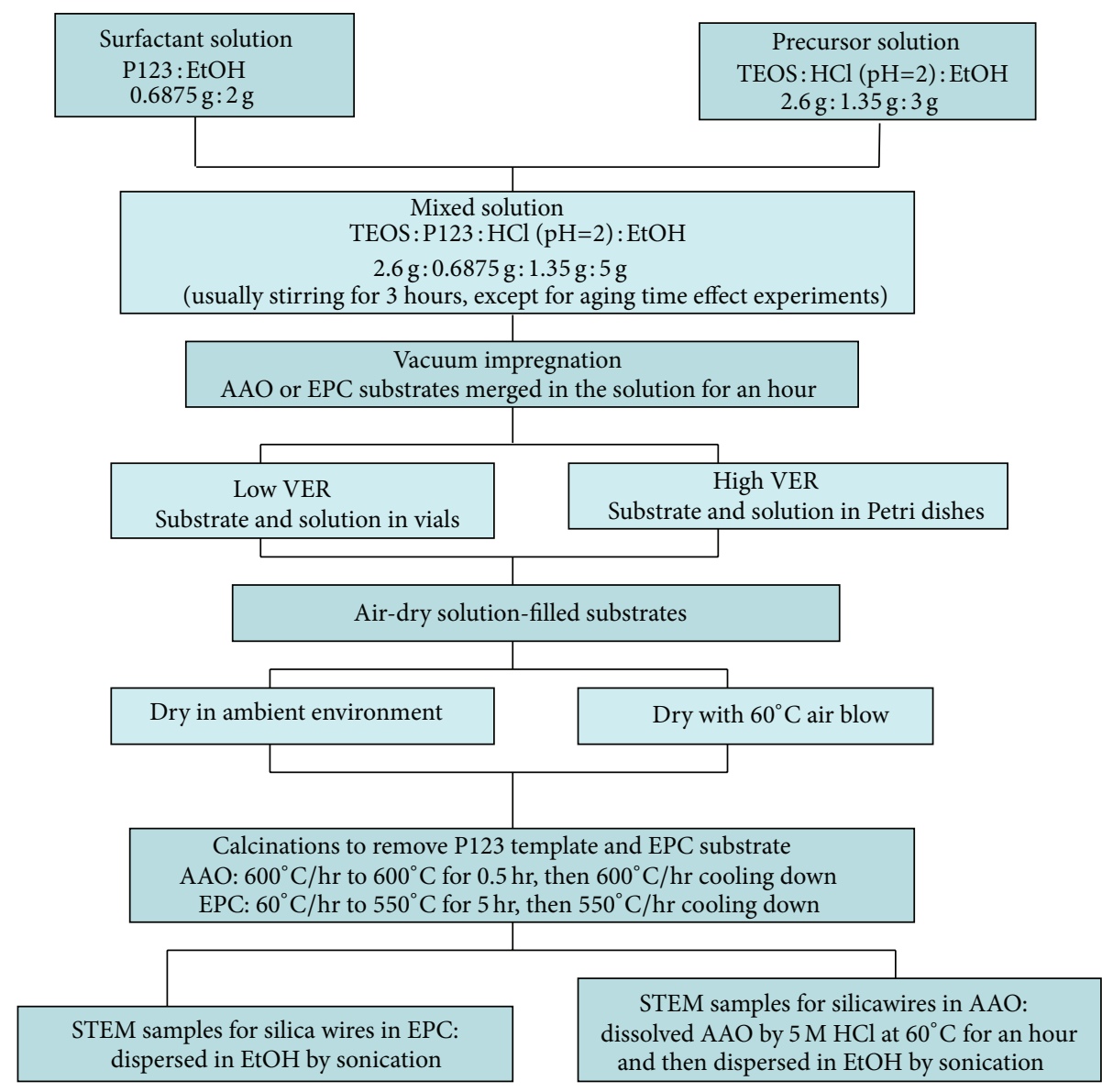

FIGURE 1: Schematic diagram showing the experimental and characterization procedures in the STEM study of the nanostructured mesoporous silica wires.

Figure 1 is the schematic diagram showing the synthetic procedure of mesoporous silica wires as well as the preparation of STEM sample grids. Two stock solutions were prepared. For the silica precursor solution, $2.6 \mathrm{~g}$ TEOS in an ethanol ( $3 \mathrm{~g}$ ) solution mixed with $1.35 \mathrm{~g}$ diluted hydrochloric acid $(\mathrm{pH} \sim 2)$ was prehydrolyzed at room temperature for typically about 10 minutes. For the templating polymer surfactant solution, ca. $0.69 \mathrm{~g}$ triblock copolymer P123 was dissolved in $2 \mathrm{~g}$ ethanol for at least 30 minutes to become homogeneous at molecular level. Afterwards, the copolymer ethanol solution was added into the acidic TEOS ethanol solution, and the mass ratio of the mixed solution was 2.6 TEOS : $0.6875 \mathrm{P} 123: 1.35 \mathrm{HCl}(0.01 \mathrm{M}, \mathrm{pH} \sim 2): 5 \mathrm{EtOH}$. This resulting mixture was stirred at room temperature $\left(\sim 22^{\circ} \mathrm{C}\right)$ for three hours. However, for some experiments, the mixture was stirred for $0.03,1.5,3$, and 48 hours at room temperature. These will be later referred as TEOS aging time experiments.

The mixed solution was loaded into the substrate AAO or EPC channels by vacuum impregnation with the substrate membranes submerged into the mixed solution for one hour at room temperature $\left(\sim 22^{\circ} \mathrm{C}\right)$. The channels-loaded AAO or EPC membranes were subsequently ambient air-dried overnight or hot air-dried. Both glass vials (about $40 \mathrm{~mL}$ with 1.37-in diameter opening) and plastic Petri dishes (about 2-in diameter opening) were used as solution containers for the loading of the mixture into the membrane channels. Two solution evaporation rates (VER: high and low) under vacuum condition were achieved, due to the significant difference in the diameter of the liquid-air interface between the vials and dishes. The relative VER value was measured, with the dish-to-vial VER ratio of ca. 1.46. Regarding the air drying after vacuum impregnation, it was carried out either under the ambient condition or at $60^{\circ} \mathrm{C}$ with air blowing by a hair dryer. The condition of the air drying process could have some impacts on the intrawire structure of the silica/P123 wire.

In the last step to get the wires out of the matrices of membrane channels, the copolymer template P123 and EPC membrane were removed by calcination at $60^{\circ} \mathrm{C} / \mathrm{h}$ to $550^{\circ} \mathrm{C}$ for 5 hours. The AAO membrane was dissolved by $5 \mathrm{M} \mathrm{HCl}$ at $60^{\circ} \mathrm{C}$ for approximately one hour or by calcination at $600^{\circ} \mathrm{C} / \mathrm{h}$ to $600^{\circ} \mathrm{C}$ for 0.5 hour.

2.3. Characterization. To prepare the specimen for scanning transmission electron microscopy (STEM), the freestand mesoporous silica wires were redispersed in $\mathrm{EtOH}$ with ultrasonication for 1-2 minutes. One drop of the wire 


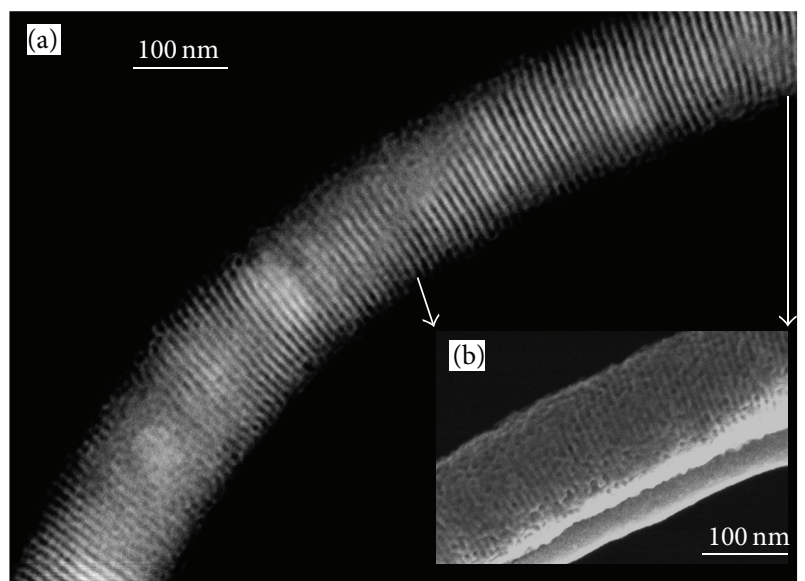

FIgURE 2: (a) A STEM image (Z-contrast mode) of one silica wire containing mesostructures of parallel-packed lamellar discs perpendicular to the wire axis. (b) A STEM image (SE mode) to reflect the surface morphology of the same wire.

suspension was then deposited onto a TEM copper grid (holy carbon-coated), followed by air drying at room temperature for at least $1 \mathrm{~h}$ before STEM examination. For each sample grid loaded with the prepared silica wires, at least three different observing spots were randomly chosen, with eight to ten STEM images taken per spot to study the intrawire morphology and structure of the mesoporous silica wires. Both volume percentage and the size of the mesopores were estimated by the STEM image evaluation.

\section{Results and Discussion}

3.1. Nanostructured Mesoporous Silica Wires with ParallelPacked Lamellae Perpendicular to the Wire Axis. Figure 2(a) shows a typical STEM image (Z-contrast transmission mode) of the mesoporous silica wire with its intrawire structure of parallel-packed lamellar "discs" perpendicular to the wire axis. This mesoporous silica wire was synthesized in $100 \mathrm{~nm}$ EPC channels with the processing condition of $3 \mathrm{~h}$ TEOS aging time, high VER, and air drying in an ambient environment. The scanning mode image (Figure $2(\mathrm{~b})$ ) shows the surface morphology of the same silica wire, indicating the possible intrawire structure of parallel-packed discs with a circular orientation. These stacked layers of discs are typically characterized by being perpendicular to the long axis of the wire. A previously published P123/water/ethanol ternary phase diagram [15] for bulk solution system suggested a possible mixture of multiple phases consisting of a cubic structure, the 3D hexagonal close packed spheres (HCPS), a 2D hexagonal, and lamellar. However, in Figure 2(a), the sideview of the porous silica wire clearly indicates a single phase of parallel-packed lamellae. As can also be seen in Figure 2(a), the packed lamellar discs exhibit a uniform interspacing. A careful evaluation based on several STEM images showed that the silica-layer thickness is on the order of $5 \mathrm{~nm}$ to $7 \mathrm{~nm}$ and the edge-to-edge distance between two nearest neighboring silica layers is $2.5 \mathrm{~nm}$ to $4.5 \mathrm{~nm}$. Note that the perpendicular lamellar structure was also observed for wires from $200 \mathrm{~nm}$ EPC channels $(1.5 \mathrm{~h})$, as shown later in Figure 5; however, those lamellar structures are not as ordered and oriented as the one in $100 \mathrm{~nm}$ EPC.

It was found that the orientation of parallel-packed discs had significant processing condition dependence. As shown in Figure 3, some of these discs have different angles to the wire axis instead of perpendicular. However, the lamellar orientation appears to have no influence on the silica-layer thickness as well as the interlayer spacing. It was also found that there is a close relationship between the orientation of the lamellar and the 2D hexagonal cylinder arrays as the former actually transforms from the latter. Thus, it is essential to establish the relationship between the disc orientations and the processing conditions for both fundamental understanding of the basic operational mechanism and structural control in the materials development.

\subsection{Synthetic Conditions Affecting the Intrawire Structures.} Three synthetic conditions are addressed in the sequence of the synthetic procedure shown in Figure 1. Typically, $50 \mathrm{~nm}$ and $200 \mathrm{~nm}$ EPC membranes were used in the TEOS aging experiments and air-drying experiments. The selection of a relatively small and a relatively large channel diameters is to see the space-confinement effect (if any) from 50 to $200 \mathrm{~nm}$ scale).

3.2.1. TEOS Aging Time. As indicated in the Experimental Section, the mixture of the two stock solutions was stirred at room temperature. The stirring period for the mixture solution is referred to as TEOS aging time, during which the early-stage hydrolysis and condensation reaction of TEOS start to occur. This aging affects the nucleation and growth rate of amorphous silica solid formation and distribution in the hydrophilic microphases of the block copolymer. The silica solid will take the mesostructure patterns of the blockcopolymer microphases. EPC membranes of $50 \mathrm{~nm}$ and $200 \mathrm{~nm}$ in channel sizes are used for this study, respectively.

Figure 4 shows the STEM images of the porous silica wires prepared in the $50 \mathrm{~nm}$ EPC channels. Corresponding to Figures $4(\mathrm{a})$ to $4(\mathrm{~d})$, the TEOS aging times are $0.03,1.5$, 3 , and 48 hours, respectively. The STEM images indicate that, for all these aging times, the dominant phase in the nanowires has worm-like feature, with a hint of hexagonal pattern. A detailed STEM examination shows that the major structure is the parallel discs for the aging time of 0.03 hours (Figure 4(a)), while much less structured silica solid is observed when aging time extends to longer time such as 48 hours (Figure $4(\mathrm{~d})$ ). Less aged solution (Figure 4(a)) indicates that lamellar mesostructure forms first in the time course of polymer microphase separations, prior to its transformation to worm-like or hexagonal structures. On the other hand, long aged solution (Figure 4(d)) seems to kill the selfassembly structure formation; most probably the microphase evolution of the polymer is "frozen" by the rapidly condensed silica solid material. Thus, near amorphous materials are formed inside wires. 


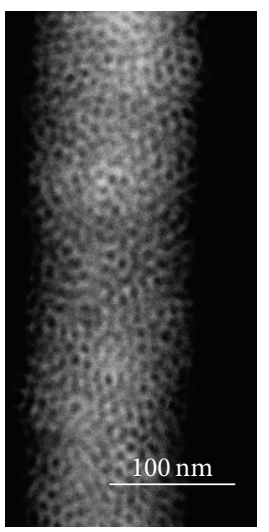

(a)

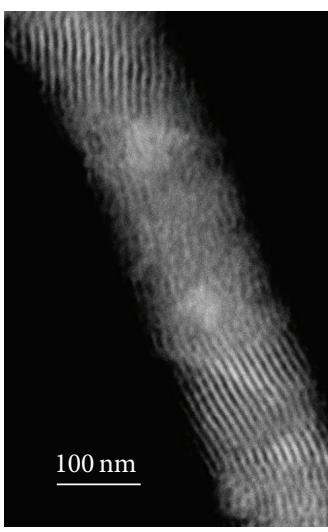

(b)

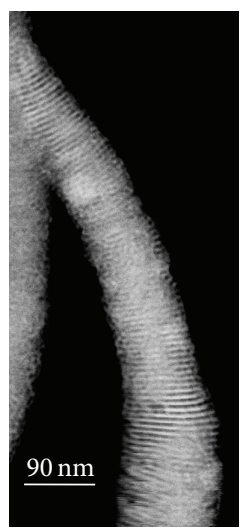

(c)

FIGURE 3: Z-contrast mode STEM images showing (a) the worm-like silica nanowires (formed in $100 \mathrm{~nm}$ EPC under low VER) with a hint of hexagonal pattern and ((b) and (c)) the parallel structure of silica nanowires (formed in $100 \mathrm{~nm}$ EPC under high VER). Note that these parallel lamellar structures have different orientation.
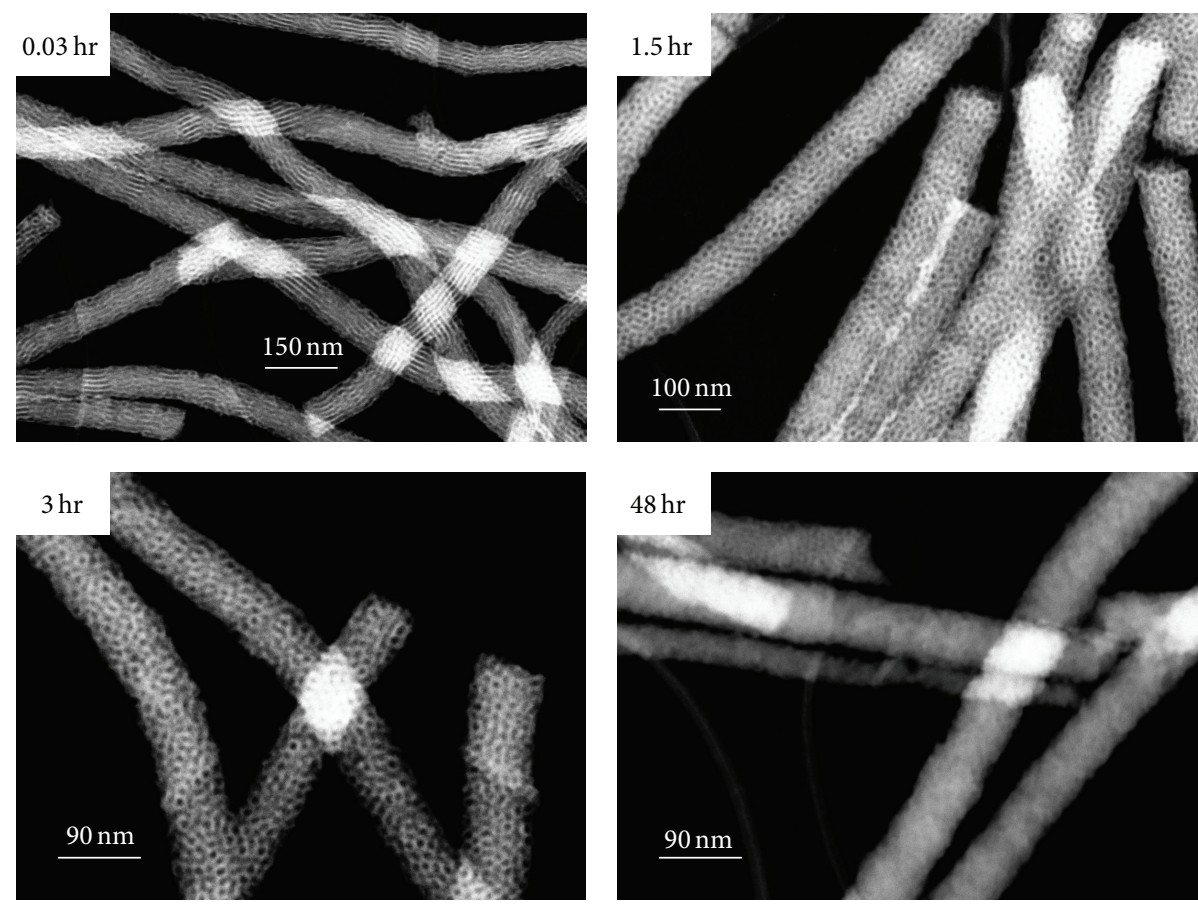

FIGURE 4: Representative STEM images (Z-contrast mode) of the structures in silica wires formed in 50 nm channels of EPC at various aging times indicated.

The same TEOS aging time experimental scheme was also carried out with the $200 \mathrm{~nm}$ EPC channels and the results are shown in Figure 5. A similar trend of structure changes with aging time was observed, however, with most of the lamellar structures formed at aging time of 1.5 hours. This indicates that the structural evolution kinetics has a dependence on the channel diameter. The aging time is significantly shorter, only 0.03 hours for self-assembly parallel disc formation inside a smaller channel dimension of $50 \mathrm{~nm}$ as indicated in Figure 4. The "critical" time for parallel disc evolution is prolonged to 1.5 hours in the larger $200 \mathrm{~nm}$ channels (Figure 5). In other words, the space confinement by the smaller channels seems to make faster of the self-assembly evolution of the silica porous mesostructures.

The self-assembly process via microphase evolution of triblock copolymer P123 plays a key role in the final nanostructure of silica wires near the critical time. Complying of silica to the polymer's microphase templating often is hindered by the condensation of TEOS after self-assembly of lamellar. The packed lamellar perpendicular to the wire axis (as shown in Figure 2) was observed in multiple occasions, such as in the silica nanowires in the $200 \mathrm{~nm}$ EPC with 1.5 hour aging time (Figure 5(b)), in $50 \mathrm{~nm}$ EPC with 0.03 hour aging time, at low VER (Figure 3(b)), and air-drying in ambient environment. 

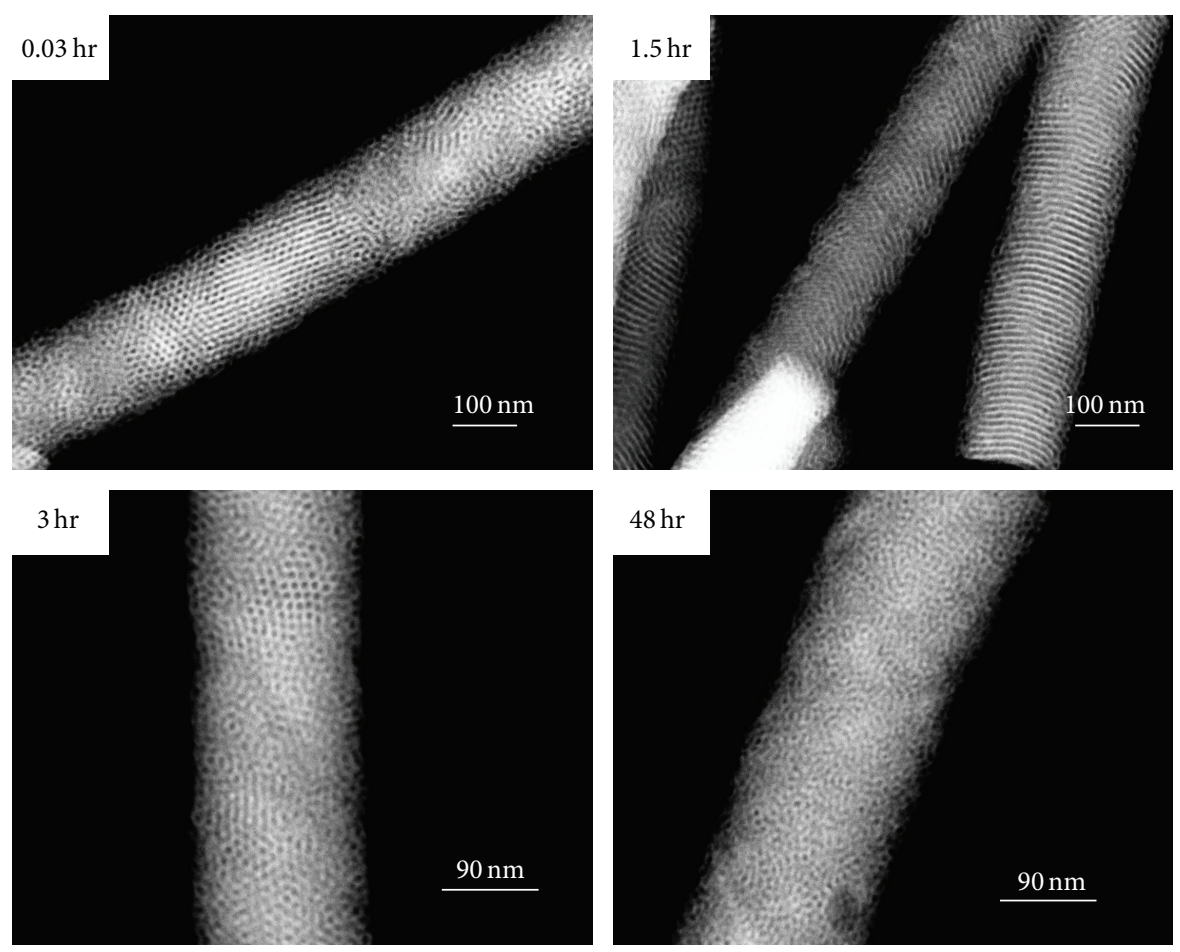

FIGURE 5: Representative STEM images (Z-contrast mode) of mesoporous structures in the silica wires formed in $200 \mathrm{~nm}$ channels of EPC for TEOS aging times indicated.

The microphase structure evolution of the triblock copolymer was hindered or even immobilized by the sol-gel reaction (and silica solid phase forming) process of inorganic species. The final polymer-silica composite self-assembly structure is determined by the timing between equilibrium/dynamics of P123 microphase formation (induced by vacuum evaporation) and sol-gel reaction kinetics of the silica precursor (affected by aging time, as well as vacuum evaporation). Also, the mechanical rigidness of the formed silica solid material could hinder or even "freeze" the transient polymer microphase, thus interfering with the normal pure polymer microphase evolution process.

In summary, the prolonged aging time effectively increases the hydrolytically condensed nanoparticle size of the silica sol/gel components in the solutions which are used to impregnate the substrate channels. The increased silica component leads to enhanced steric hindrance to the self-assembly of the P123-silica hybrid mesophases. Another factor that increases the steric hindrance effect is significant reduction in the dimension of the substrate channels (i.e., space confinement effect).

3.2.2. VER Effect. The VER represents the evaporation rate of the bulk solution of P123-silica precursor mixture used for impregnation of substrate channels under vacuum condition. However, the solution soaked inside the substrate channels may experience a different evaporation rate. Nonetheless, the bulk evaporation rate is directly correlated to the inchannel evaporation rate. As discussed above, a packed lamellar disc structure (Figure 2(a)) was observed in the silica wires produced by the $100 \mathrm{~nm}$ EPC under high VER, but none of which was observed in the low VER. Instead, for low VER, the worm-like morphology with the hint of hexagonal pattern (Figure 3(a)) is the dominating feature in the intrawire mesostructures.

The parallel structure (lamellar phase or hexagonal packed cylinder phase) develops preferentially under high VER for all silica wires in the $100 \mathrm{~nm}$ EPC, although with varied orientations, that is, either normal or parallel to the long wire axis, or having an angle to it (Figures 3(b) and 3(c)). It is quite possible that higher VER produced a suitable timing for the P123 self-assembled into parallel structure without being hindered by the silica components. Under lower VER, however, the silica species already condensed large enough to hinder the microphase transformation, resulting in a wormlike structure with hint of hexagonal pattern. There are only slight intrawire structural differences among those formed under high VER and low VER in $50 \mathrm{~nm}$ EPC, $200 \mathrm{~nm}$ EPC, and $200 \mathrm{~nm}$ AAO. This behavior may be due to the substrate channel confinement of the critical sizes. VER effect on intrawire nanostructure is also altered by the nanoscale channel confinement, which is a diffusion governing parameter. Thus, VER is a combination effect influenced by solvent evaporation, channel size, and the condensed size of silica components.

3.2.3. Air-Dry Condition Effect. As shown in the procedure flowchart, air-drying was performed immediately after the vacuum impregnation.

The effect was investigated on the silica nanowires in $50 \mathrm{~nm} \mathrm{EPC,} 200 \mathrm{~nm} \mathrm{EPC}$, and $200 \mathrm{~nm}$ AAO. Based on the results of STEM images, under $60^{\circ} \mathrm{C}$ air blow drying 


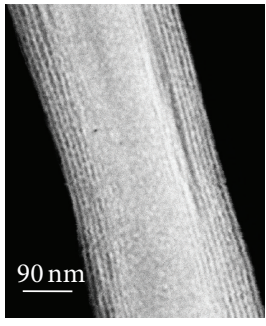

(a)

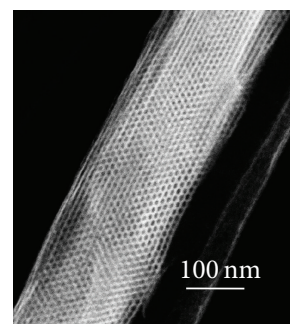

(b)

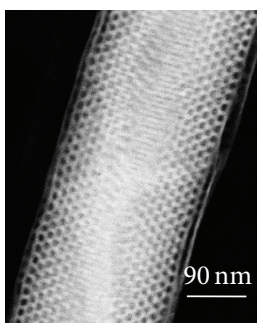

(c)

FIgURE 6: Representative STEM images (Z-contrast mode) showing the structures of silica wires in $200 \mathrm{~nm}$ AAO under $60^{\circ} \mathrm{C}$ air blow drying condition: (a) parallel lamellar planes along the axis, (b) parallel-packed channels perpendicular to the wire axis, and (c) circular channels in the axial direction.

condition, the parallel structure is preferentially developed in the silica wires produced in both $50 \mathrm{~nm}$ EPC and $200 \mathrm{~nm}$ EPC channels. It should be noted that, according to Brinker and Scherer [16], at a given vapor pressure, larger pores dry faster because all menisci have the same curvature. Thus, substrate channels with larger diameters imply faster solvent evaporation. For the lamellar structure of silica wires in $200 \mathrm{~nm}$ EPC, there is no significant space distance difference between ambient air-drying and $60^{\circ} \mathrm{C}$ air blow drying.

For the same diameter channels, AAO shows a different behavior from the EPC regarding air-drying effect on mesostructure formation in wires. STEM image in Figure 6(a) indicates a small volume fraction ( 1 vol. $\%)$ with parallel-lamellar-planes aligned with the wire axis. About 5 vol.\% of the parallel-packed channel structure with the channels perpendicular to the wire axis is observed in the $200 \mathrm{~nm}$ AAO, under $60^{\circ} \mathrm{C}$ air blow drying, as shown in Figure $6(\mathrm{~b})$. The average pore size of the circular-channel structure (Figure 6(c)) is $6.2 \mathrm{~nm}$ in silica wires from the $200 \mathrm{~nm}$ AAO, which is smaller than that by ambient airdrying, (average of $10.6 \mathrm{~nm}$ ). Therefore, it appears that the drying condition has a pronounced effect on both the structure and the pore size in the $200 \mathrm{~nm}$ AAO channels.

\section{Conclusions}

A new intrawire nanostructure, namely, lamellar disc, perpendicular to the wire axis, in silica nanowires is reported here via confinement in both AAO and EPC channels. The processing conditions are varied to achieve different structural features and lamellar orientations. These conditions include preassembly aging time, vacuum evaporation rate, and air-drying environment. It has been found that the intrawire nanopore structure is sensitive to the processing conditions. Furthermore, the nanoscale confinement is also an important parameter as it dictates the diffusion properties. Therefore, in the P123-TEOS-water-EtOH quaternary system, the nanostructure formation is the result of synergism of the block copolymer and silica components cooperative molecular self-assembly, affected by processing conditions and the nanoscale space confinement. Based on the findings in this study, it is to design and develop desired intrawire nanostructure by controlling the processing conditions and the substrate characteristics.

\section{Conflict of Interests}

The authors declare that there is no conflict of interests regarding the publication of this paper.

\section{Acknowledgments}

The authors acknowledge Peng Lai for her involvement of conducting some hands-on experiments and discussions during her postmaster work at the Oak Ridge National Laboratory (ORNL). This work on nanostructure control inside the nanochannel array membranes is partially supported by the ORNL LDRD-SEED Money Program Fund, partially by the DOE/EERE/BETO Program Fund, and partially by the Division of Materials Science (KC 0203010 ), Office of Science, the U.S. Department of Energy. The STEM characterization was sponsored in part by the Assistant Secretary for Energy Efficiency and Renewable Energy, Office of Transportation Technologies, as part of the High Temperature Materials Laboratory, managed by UT-Battelle, LLC, for the U.S. Department of Energy under contract DE-AC0500OR22725.

\section{References}

[1] C. Sanchez, B. Julián, P. Belleville, and M. Popall, "Applications of hybrid organic-inorganic nanocomposites," Journal of Materials Chemistry, vol. 15, no. 35-36, pp. 3559-3592, 2005.

[2] Y. Wu, T. Livneh, Y. X. Zhang et al., "Templated synthesis of highly ordered mesostructured nanowires and nanowire arrays," Nano Letters, vol. 4, no. 12, pp. 2337-2342, 2004.

[3] G. S. Attard, J. C. Glyde, and C. G. Göltner, "Liquid-crystalline phases as templates for the synthesis of mesoporous silica," Nature, vol. 378, no. 6555, pp. 366-368, 1995.

[4] C. G. Göltner, S. Henke, M. C. Weissenberger, and M. Antonietti, "Mesoporous silica from lyotropic liquid crystal polymer templates," Angewandte Chemie, vol. 37, no. 5, pp. 613-616, 1998.

[5] C. J. Brinker, Y. Lu, A. Sellinger, and H. Fan, "Evaporationinduced self-assembly: nanostructures made easy," Advanced Materials, vol. 11, no. 7, pp. 579-585, 1999.

[6] X. Ren and Z. Lun, "Mesoporous silica nanowires synthesized by electrodeposition in AAO," Materials Letters, vol. 68, pp. 228-229, 2012.

[7] Z. Gong, G. Ji, M. Zheng et al., "Structural characterization of mesoporous silica nanofibers synthesized within porous 
alumina membranes," Nanoscale Research Letters, vol. 4, no. 11, pp. 1257-1262, 2009.

[8] K. J. Lee, S. H. Min, and J. Jang, "Mesoporous nanofibers from dual structure-directing agents in AAO: mesostructural control and their catalytic applications," Chemistry, vol. 15, no. 11, pp. 2491-2495, 2009.

[9] P. Lai, M. Z. Hu, D. Shi, and D. Blom, "STEM characterization on silica nanowires with new mesopore structures by spaceconfined self-assembly within nano-scale channels," Chemical Communications, no. 11, pp. 1338-1340, 2008.

[10] B. Platschek, N. Petkov, and T. Bein, "Tuning the structure and orientation of hexagonally ordered mesoporous channels in anodic alumina membrane hosts: a $2 \mathrm{D}$ small-angle X-ray scattering study," Angewandte Chemie, vol. 45, no. 7, pp. 1134$1138,2006$.

[11] B. Yao, D. Fleming, M. A. Morris, and S. E. Lawrence, "Structural control of mesoporous silica nanowire arrays in porous alumina membranes," Chemistry of Materials, vol. 16, no. 24, pp. 4851-4855, 2004.

[12] G. Kickelbick, "Formation of hexagonal mesoporous silica in submicrometer channels," Small, vol. 1, no. 2, pp. 168-170, 2005.

[13] J. Wang, C.-K. Tsung, W. Hong, Y. Wu, J. Tang, and G. D. Stucky, "Synthesis of mesoporous silica nanofibers with controlled pore architectures," Chemistry of Materials, vol. 16, no. 24, pp. 51695181, 2004.

[14] Z. Liang and A. S. Susha, "Mesostructured silica tubes and rods by templating porous membranes," Chemistry, vol. 10, no. 19, pp. 4910-4914, 2004.

[15] S. S. Soni, G. Brotons, M. Bellour, T. Narayanan, and A. Gibaud, "Quantitative SAXS analysis of the P123/water/ethanol ternary phase diagram," Journal of Physical Chemistry B, vol. 110, no. 31, pp. 15157-15165, 2006.

[16] C. J. Brinker and G. W. Scherer, SOL-GEL Science, Academic Press, San Diego, Calif, USA, 1990. 

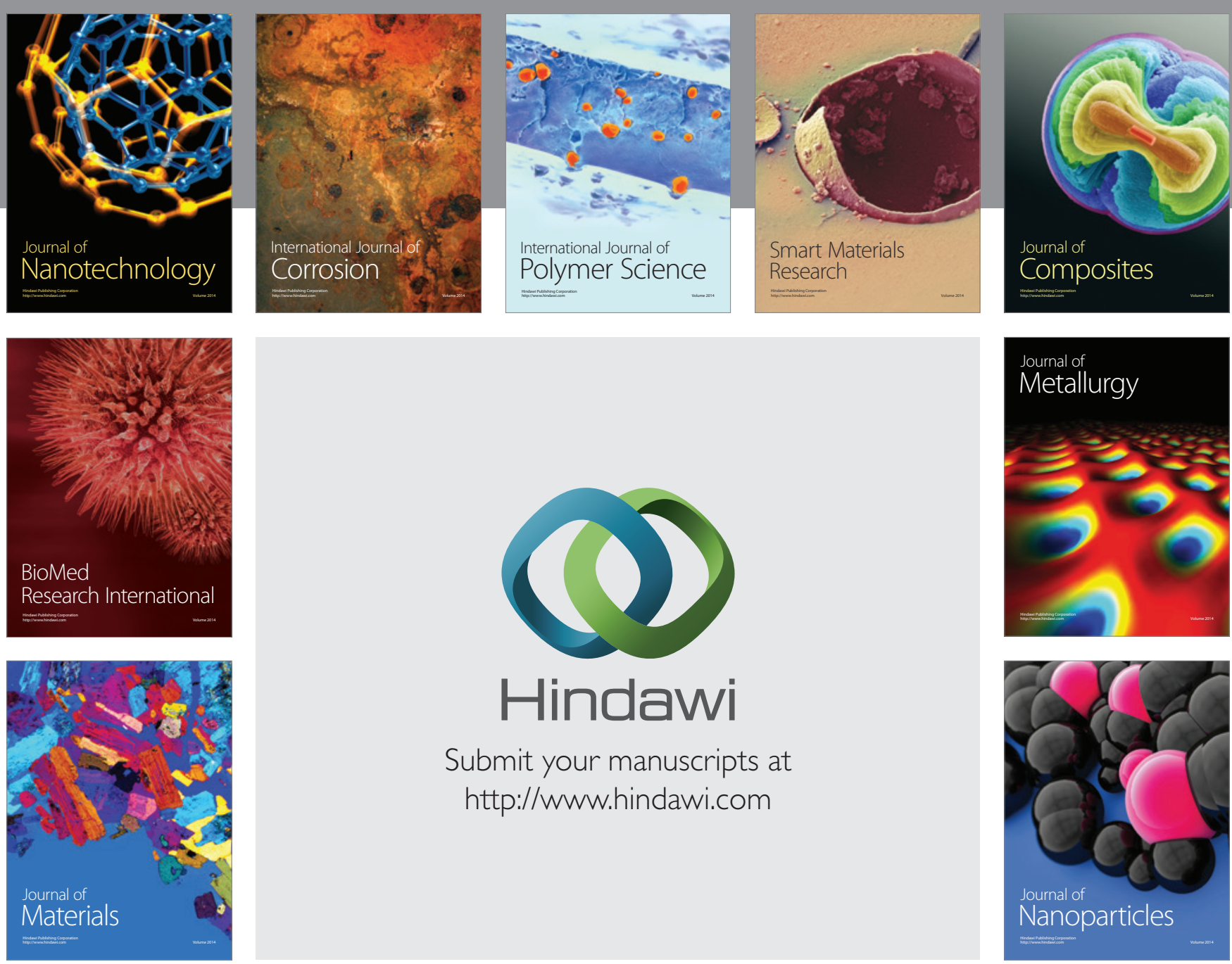

Submit your manuscripts at http://www.hindawi.com
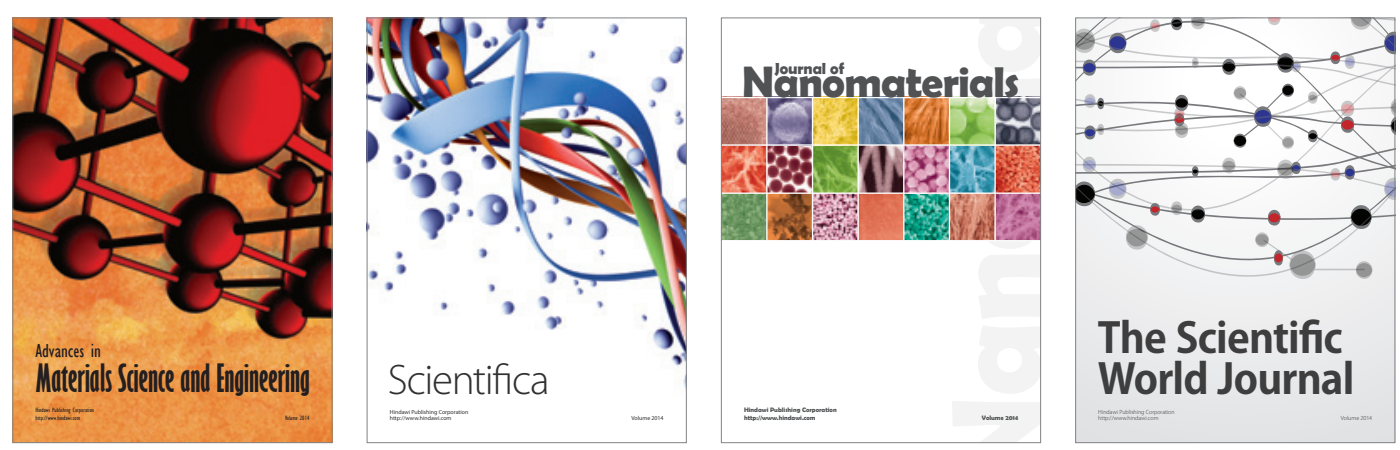

\section{The Scientific World Journal}
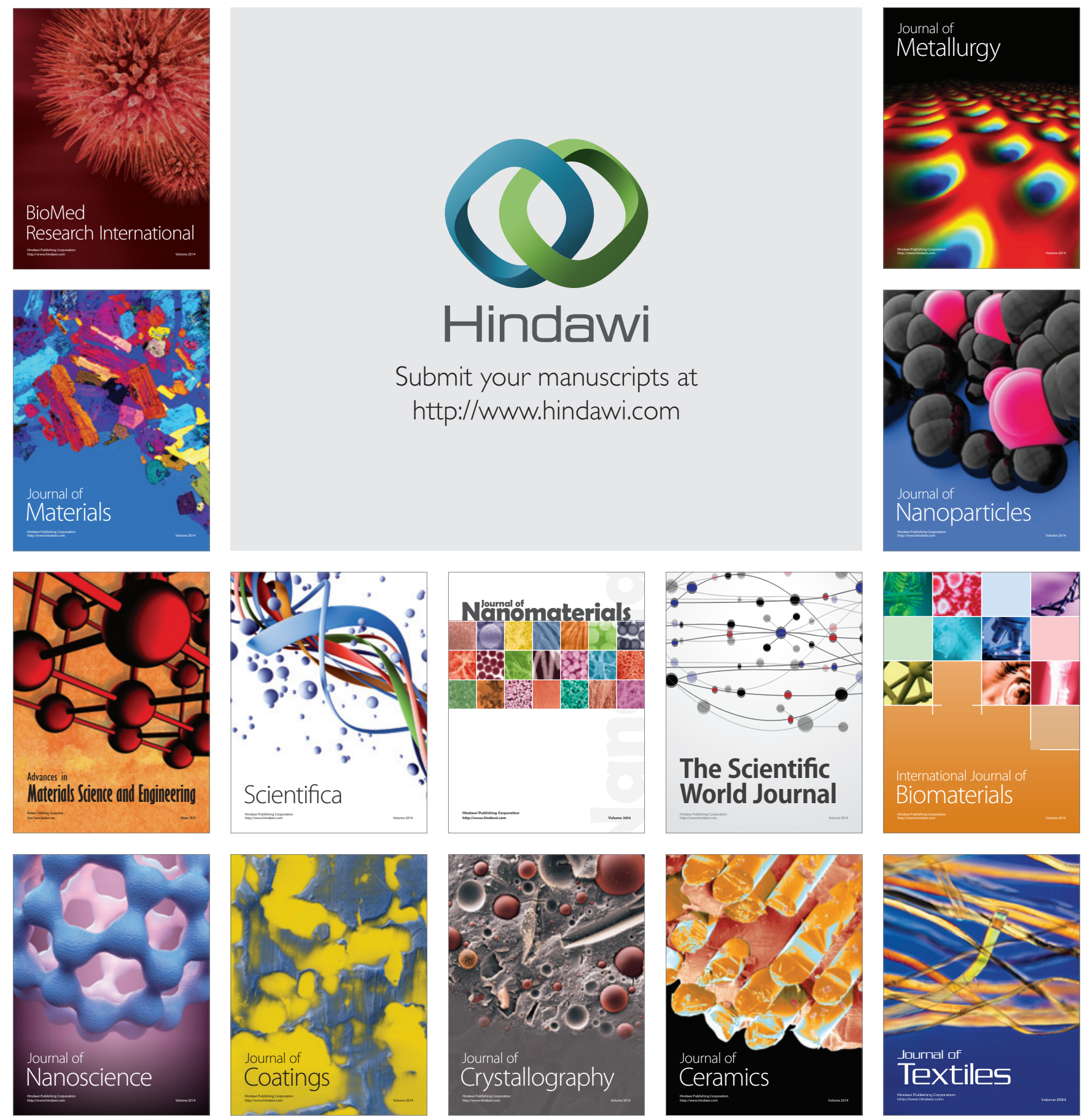